\title{
Mutational Evolution of Pseudomonas aeruginosa Resistance to Ribosome-Targeting Antibiotics
}

\author{
Fernando Sanz-García, Sara Hernando-Amado* and José L. Martínez* \\ Centro Nacional de Biotecnología, Consejo Superior de Investigaciones Científicas, Madrid, Spain
}

The present work examines the evolutionary trajectories of replicate Pseudomonas aeruginosa cultures in presence of the ribosome-targeting antibiotics tobramycin and tigecycline. It is known that large number of mutations across different genes - and therefore a large number of potential pathways - may be involved in resistance to any single antibiotic. Thus, evolution toward resistance might, to a large degree, rely on stochasticity, which might preclude the use of predictive strategies for fighting antibiotic resistance. However, the present results show that $P$. aeruginosa populations evolving in parallel in the presence of antibiotics (either tobramycin or tigecycline) follow a set of trajectories that present common elements. In addition, the pattern of resistance mutations involved include common elements for these two ribosome-targeting antimicrobials. This indicates that mutational evolution toward resistance (and perhaps other properties) is to a certain degree deterministic and, consequently, predictable. These findings are of interest, not just for $P$. aeruginosa, but in understanding the general rules involved in the evolution of antibiotic resistance also. In addition, the results indicate that bacteria can evolve toward higher levels of resistance to antibiotics against which they are considered to be intrinsically resistant, as tigecycline in the case of $P$. aeruginosa and that this may confer cross-resistance to other antibiotics of therapeutic value. Our results are particularly relevant in the case of patients under empiric treatment with tigecycline, which frequently suffer $P$. aeruginosa superinfections.

Keywords: antibiotic resistance, Pseudomonas aeruginosa, tobramycin, tigecycline, mutation, evolution

This article was submitty section

Evolutionary and Genomic Microbiology,

a section of the journal

Frontiers in Genetics

Received: 26 July 2018

Accepted: 18 September 2018

Published: 18 October 2018

Citation:

Sanz-García F

Hernando-Amado S and Martínez JL (2018) Mutational Evolution of Pseudomonas aeruginosa Resistance to Ribosome-Targeting Antibiotics. Front. Genet. 9:451. doi: 10.3389/fgene.2018.00451

\section{INTRODUCTION}

Antibiotic resistance has been a major public health concern since the dawn of the antibiotic era, but in recent decades there has been an alarming increase in the number and type of antibiotic-resistant bacteria (Appelbaum, 2012), posing a threat to health worldwide (Roca et al., 2015). Predicting the mechanisms by which bacteria may acquire resistance is therefore important in the prevention and treatment of infections (Martinez et al., 2007; Martinez et al., 2011).

Resistance can be acquired via horizontal transfer of antibiotic resistance genes (HGT), or through mutation (Hernando-Amado et al., 2017). While exhaustive information is available on the mechanisms of antibiotic resistance at the basic science and epidemiological levels, the evolutionary trajectories leading to high level antimicrobial resistance, as well as the reproducibility of these trajectories among populations evolving concurrently, have been studied in less detail. It is worth mentioning, however, that the reconstruction of mutants that are selected in patients 
under treatment have shown that fitness costs and the selection of compensatory mutations are critical for the success of some specific antibiotic resistance mutations (Shcherbakov et al., 2010; Brandis et al., 2015; Meftahi et al., 2016; Huseby et al., 2017). Nevertheless, this type of retrospective analyses is useful just for studying already known mechanisms of resistance, not for predicting new ones (Pietsch et al., 2017).

Strategies to predict the emergence of resistance mutations (Martinez et al., 2007) were implemented soon after the discovery of antibiotics (Bryson and Szybalski, 1952), one of the most useful of which is experimental evolution. Since the seminal work of Richard Lenski, experimental evolution has been used to analyze different bacterial traits, including the development of resistance to antibiotics (Bryson and Szybalski, 1952; Toprak et al., 2011; Turrientes et al., 2013; Feng et al., 2016; Ibacache-Quiroga et al., 2018). Recent research has shown experimental evolution able to predict the emergence of resistance against different antimicrobial agents, including colistin (Jochumsen et al., 2016), beta-lactams, quinolones and aminoglycosides (Cabot et al., 2016; Feng et al., 2016; Ibacache-Quiroga et al., 2018; Lopez-Causape et al., 2018b). In recent years, the potential of experimental evolution has been further boosted by the development of technologies that allow the fast and affordable sequencing of whole bacterial genomes.

Pseudomonas aeruginosa, an opportunistic pathogen widely distributed in nature (Silby et al., 2011), commonly causes lung, airway and other infections in hospitalized patients. It is the main cause of chronic infections in patients with cystic fibrosis (CF) and chronic obstructive pulmonary disease (MartinezSolano et al., 2008; Tummler et al., 2014). These infections are usually fought using aminoglycosides, $\beta$-lactams and polymyxins (Palmer and Whiteley, 2015). Unfortunately, P. aeruginosa intrinsically shows low-level susceptibility to a number of drugs, even against the recently developed glycylcycline tigecycline (Pankey, 2005), which works via tightly binding to the ribosome and thus evading the most common tetracycline resistance mechanisms. In addition, mutants presenting increased levels of antibiotic resistance are selected along chronic infections. Indeed, while resistance to aminoglycosides in $P$. aeruginosa isolates from acute infections has been largely attributed to the acquisition of antibiotic resistance genes, mutation plays a major role for the acquisition of resistance by $P$. aeruginosa causing chronic infections (Vogne et al., 2004; Guenard et al., 2014; Bolard et al., 2018).

In the present work, experimental evolution and wholegenome sequencing (WGS) were used to examine evolutionary trajectories of $P$. aeruginosa toward resistance against two ribosome-targeting antimicrobials: tobramycin and tigecycline. The aim was to determine whether the mechanisms that impair the actions of different drugs targeting the same cell machinery are shared (at least in part), or whether resistance to each drug is specific via a different mechanism. Tigecycline binds to the $30 \mathrm{~S}$ ribosomal subunit, thereby blocking the interaction of aminoacyl-tRNA with the A site of the ribosome, whereas tobramycin prevents the formation of the 70S complex (Kotra et al., 2000). While tobramycin forms part of usual therapy regimens against $P$. aeruginosa (Cheer et al., 2003), the pathogen is intrinsically resistant to tigecycline (following the clinical definition of antibiotic resistance) (Martinez et al., 2015). One cause of this phenotype is the capability of the multidrug efflux pump MexXY, also involved in intrinsic $P$. aeruginosa tobramycin resistance (Westbrock-Wadman et al., 1999), to extrude tigecycline (Dean et al., 2003). Nonetheless, tigecycline was used in the present study since it provides an opportunity to examine whether or not bacterial pathogens can acquire clinically relevant characteristics when challenged with the antibiotics to which they are considered to be intrinsically resistant. In this regard, it is worth mentioning that $P$. aeruginosa has emerged as a major cause of superinfection in nosocomial patients treated with tigecycline (Garcia-Cabrera et al., 2010; Ulu-Kilic et al., 2015; Katsiari et al., 2016). Knowing whether or not the empirical use of tigecycline for treating Gramnegative hospital infections might challenge $P$. aeruginosa, affecting its susceptibility to other antibiotics commonly used for treating $P$. aeruginosa infections, is of relevance for developing a rational approach for treating such superinfections. The results showed that even for microorganisms dubbed intrinsically resistant to an antibiotic, the challenge with this antibiotic selects mutants presenting reduced susceptibility to antibiotics of clinical value.

Experimental evolution studies allow one to determine whether evolutionary trajectories are reproducible, i.e., whether the process of evolution is mainly deterministic and hence predictable (Martinez et al., 2007), or whether it is largely stochastic. The present work provides a predictive analysis of the potential mutational causes of resistance in $P$. aeruginosa against two ribosome-targeting antibiotics belonging to different structural families, as well as the different evolutionary trajectories taken toward this resistance. This information may allow new strategies to be designed for predicting, managing, and eventually reducing resistance in this important nosocomial pathogen. In addition, the results throw light on whether bacterial evolution is largely stochastic or presents some deterministic features, and thus whether the emergence and spread of antibiotic resistance can be predicted to a certain extent (Martinez et al., 2007, 2011).

\section{MATERIALS AND METHODS}

\section{Growth Conditions and Determination of Susceptibility to Antibiotics}

Unless otherwise stated, bacteria were grown in Mueller Hinton Broth (MHB, Pronadisa) at $37^{\circ} \mathrm{C}$ with agitation at $250 \mathrm{rpm}$. The initial concentrations of tigecycline (Pfizer) and tobramycin (Normon, S. L) that inhibit the growth of $P$. aeruginosa PA14 under the culture conditions used in the evolution experiments were determined at $37^{\circ} \mathrm{C}$.

General susceptibility to a wide range of antibiotics tigecycline, tetracycline, aztreonam, ceftazidime, imipenem, ciprofloxacin, levofloxacin, norfloxacin, tobramycin, streptomycin, amikacin, colistin, polymyxin B, chloramphenicol, fosfomycin and erythromycin - was examined by disk diffusion in Mueller Hinton Agar (MHA) (Sigma) at $37^{\circ} \mathrm{C}$. 
The MICs of different antibiotics were determined for the bacterial populations over the evolution period at $37^{\circ} \mathrm{C}$ in $\mathrm{MHA}$ using E-test strips (MIC Test Strip, Liofilchem ${ }^{\circledR}$ ). MICs of colistin and polymyxin B were determined in MHB II by double dilution in microtiter plates. The MICs to the antibiotics of selection and to fosfomycin were repeated twice and in all cases, the results were the same in the replicated assays.

\section{Experimental Evolution}

Twelve independent bacterial populations (four controls without antibiotics, four populations challenged with tigecycline, and four populations challenged with tobramycin) were grown in parallel in MHB for 35 consecutive days. All replicates were established from a stock culture of the $P$. aeruginosa PA14 strain. Each day, the cultures were diluted (1/250) with fresh MHB: $8 \mu \mathrm{l}$ of bacterial culture in $2 \mathrm{ml}$ of medium. The concentrations of tigecycline and tobramycin used for selection increased over the evolution period from the initial MIC up to 32MIC, doubling them every 5 days. Every 5 days, samples from each culture were taken and preserved at $-80^{\circ} \mathrm{C}$ for future investigation.

\section{Whole-Genome Sequencing}

Genomic DNA was extracted at the end of the evolution assays from all 12 populations using the Gnome ${ }^{\circledR}$ DNA kit (MP Biomedicals). Whole-genome sequencing was performed by Sistemas Genómicos S.L. Libraries were obtained without amplification following Illumina protocols and recommendations. The quality of the extracted material was analyzed via a 4200 Tapestation High Sensitivity assay, and the DNA concentration determined by real-time PCR using a LightCycler 480 device (Roche). The pool of libraries was pairend sequenced $(100 \times 2)$ in an Illumina HiSeq 2500 sequencer. The average number of reads per sample was 8646177 , which represents a coverage of 200x on average. Short reads used in this publication are deposited in SRA database ${ }^{1}$ with accession PRJNA490803.

\section{Bioinformatic Analysis}

Mutations in the evolved bacteria were detected using CLC Genomics Workbench 9.0 (QIAGEN) software. WGS data were trimmed and the reads aligned with the $P$. aeruginosa UCBPP-PA14 reference chromosome (NC_008463.1). The single nucleotide polymorphisms (SNPs) present in the populations kept under selective pressure were identified and filtered against those present in the populations maintained in the absence of such pressure. The cut-off threshold of a mutation to be included in the analysis was $\geq 15 \%$.

\section{Confirmation of SNPs}

Sanger sequencing was used to verify the mutations found via WGS (Supplementary Table S1) and to ascertain the order of appearance of these modifications. Twenty-four pairs of primers, which amplified 100-200 base pair regions containing each putative mutation, were designed (Supplementary Table S2). After PCR amplification, the corresponding amplicons were

${ }^{1}$ https://www.ncbi.nlm.nih.gov/sra purified using the QIAquick PCR Purification Kit (QIAGEN) and sequenced at GATC Biotech.

\section{RNA Extraction and Real-Time RT-PCR}

One flask containing $20 \mathrm{ml}$ of $\mathrm{MHB}$ was inoculated with an overnight culture of the selected clones and $P$. aeruginosa PA14 to a final O.D.600 $=0.01$, and they were incubated until exponential phase was reached (O.D.600 $=0.6$ ). $10 \mathrm{ml}$ of each culture were centrifuged at $7000 \mathrm{rpm}$ for $15 \mathrm{~min}$ and at $4^{\circ} \mathrm{C}$. This process was performed with three independent biological replicates.

Then, RNeasy mini Kit (QIAGEN) extraction protocol was followed: $570 \mu \mathrm{l}$ of TE buffer $(10 \mathrm{mM}$ Tris-HCl, $1 \mathrm{mM}$ EDTA [pH 8.0]) and $30 \mu \mathrm{l}$ of lysozyme (Sigma), for a final concentration of $1 \mathrm{mg} / \mathrm{ml}$ of the latter, were added to each sample. Afterward, the samples were mixed by vortexing for $10 \mathrm{~s}$ and were incubated at room temperature for $10 \mathrm{~min}$ with regular vortexing. A volume of $2100 \mu \mathrm{l}$ of buffer RLT (QIAGEN) was added, and samples were sonicated at $0.45 \mathrm{~Hz}$ for $20 \mathrm{~s}$. Next, $1410 \mu \mathrm{l}$ of ethanol (Merck) was added and the protocol continued according to the manufacturer's instructions. In order to remove any residual DNA, two DNase treatments were carried out, with DNase I (QIAGEN) and TURBO DNase (Ambion). Finally, a PCR with rplU primers (Supplementary Table S2) was performed to check that no residual DNA was present in the RNA samples. High Capacity cDNA Reverse Transcription Kit (Applied Biosystems) was used to synthesize cDNA from $5 \mu \mathrm{g}$ of RNA. Then, realtime RT-PCR was performed using $50 \mathrm{ng}$ of cDNA and the Power SYBR green PCR Master Mix (Applied Biosystems) in the ABI PRISM 7500 real-time PCR system (Applied Biosystems). Gene expression data were normalized using the gene rplU (Supplementary Table S2). Differences in the relative amounts of mRNA were obtained following the $2^{-\Delta \Delta \mathrm{Ct}}$ method (Livak and Schmittgen, 2001).

\section{RESULTS AND DISCUSSION}

Different publications have shown that the genes that contribute to antibiotic resistance (intrinsic resistome) comprise around 3\% of the genome of a given bacterial species (Breidenstein et al., 2008; Fajardo et al., 2008; Tamae et al., 2008; Alvarez-Ortega et al., 2010; Blake and O'Neill, 2013; Fernandez et al., 2013). In the case of $P$. aeruginosa the search of a comprehensive transposon-tagged library has shown that the inactivation of 135 genes renders lowlevel tobramycin resistance (Schurek et al., 2008). Even if we do not take into consideration gain of function mutations (which are not considered in transposon insertion libraries studies), neither that different mutations might be selectable in each of these genes (Martinez and Baquero, 2000), hence increasing the number of potential antibiotic resistance mutants (Lopez-Causape et al., 2018a), the number of possible combinations of these 135 genes is $2.7 \mathrm{E} 230$. It can be argued that the number of mutations that a single bacterium can accumulate is likely low. However, even if only five mutations are accumulated as reported in the present work (see below), the number of combinations of these 135 potential mutations, taken five by five, is $4.1 \mathrm{E} 10$ if the order of 
selection is taken into consideration and 3.4E8 if the order of selection of each of the mutations along evolution is not taken into consideration. If all possible combinations were equivalent in terms of antibiotic resistance, these numbers would mean that mutation-driven antibiotic resistance should be un-predictable.

\section{Stepwise Evolution of $P$. aeruginosa Toward Antibiotic Resistance}

To determine whether similar potential evolutionary trajectories are followed by different populations, four biological replicates were allowed to evolve in parallel under selective pressure from tobramycin (populations 1-4), tigecycline (populations 5-8) and in the absence of any selective pressure (populations 9-12). In populations $1-8$ the antibiotic concentration was doubled every 5 days, from $0.5 \mu \mathrm{g} / \mathrm{ml}$ for tobramycin and $4 \mu \mathrm{g} / \mathrm{ml}$ for tigecycline, up to $32 \mathrm{MIC}$. At $64 \mathrm{MIC}$ no growth was seen after 5 days incubation, suggesting this concentration to be beyond limits of $P$. aeruginosa when evolving toward tobramycin and tigecycline resistance in these experimental conditions.

To monitor the evolution of resistance over the selection period, the susceptibility of each population to the selecting antibiotic was determined every 5 days by E-test. When bacteria are confronted to increased concentrations of antibiotics, two different phenotypic trajectories can be foreseen. Sudden selection of high-level resistance at the first steps of evolution or stepwise selection of mutants presenting increasing resistance levels. As shown in Figure 1, a stepwise evolutionary trajectory was observed for both antibiotics, suggesting either accumulation of sequential mutations after each evolution step (i.e., each change in antibiotic concentration), or the displacement of lowlevel resistance mutants by higher-level resistance ones as the selection pressure increased. It is important to note that the evolutionary trajectory was similar in all replicated experiments (Figure 1). This suggests the number of evolutionary trajectories possible (at least at phenotypic level) is limited, although the amount of genotypic evolutionary trajectories can be larger (Lassig et al., 2017).

An increase in the MIC of an antibiotic after experimental evolution does not, however, necessarily mean that antibioticresistant mutants have been selected for: resistance may be due to a phenotypic adaptation to the presence of an antibiotic rather than to mutations (Levin and Rozen, 2006; Martinez et al., 2009; Martinez and Rojo, 2011). To address this possibility, the evolved populations were sub-cultured in the absence of selection pressure (three sequential passages in MHB) and the MICs again determined. These were found not to change, indicating that the observed modifications were mainly due to the selection of stable mutants.

\section{Cross-Resistance and Collateral Sensitivity of Evolved Populations}

To determine whether the development of resistance was specific to the selecting antibiotic or also affected susceptibility to other antimicrobials, a range of representative antibiotics was tested (beta-lactams, quinolones, tetracyclines, macrolides, aminoglycosides, polymyxins, and chloramphenicol) by disk diffusion (Supplementary Table S4). Despite it has been shown macrolides as azithromycin can select mexCD-oprJ overpressing mutants in $P$. aeruginosa biofilms (Mulet et al., 2009), no differences in susceptibility to imipenem and erythromycin were detected between the wild-type parental $P$. aeruginosa PA14 strain and the evolved populations. Whether these differences can be due to the different experimental model (biofilm or planktonic cells) or the different genetic background where mutants are selected (PAO1 or PA14) remains to be established. Nevertheless, almost every evolved population developed resistance against other antibiotics belonging to the different structural families, implying that at least some resistance mutations are not tigecycline- or tobramycin-specific (Table 1). Notably, in all cases the evolved populations were more susceptible to fosfomycin than the wild-type strain, and it is important to know whether this is related to the development of resistance to ribosometargeting agents. In this regard, it is worth mentioning that Listeria monocytogenes is more susceptible to fosfomycin when growing intracellularly than when growing extracellularly. The reason for this lies in the overexpression of a hexose-phosphate transporter when the bacteria are grown intracellularly, which provides an entry route for this antibiotic (Scortti et al., 2006). These results indicate that the resistance to ribosome-targeting drugs may correlate with increasing susceptibility to fosfomycin, although the underlying mechanisms remain obscure. Given that these types of drug are widely used clinically, it is important to determine whether this trade-off occurs commonly, and whether the combined or sequential use of both types of antibiotic offers a better alternative to current therapies.

\section{Mutations Selected in the Presence of Antibiotics}

To gain insight into the genetic events associated with the development of resistance in the evolved populations, the genomes of each, as well as that of the original population (for which a frozen sample was available), were sequenced on the last day of the experiment (when the antibiotic concentration was 32MIC). Different mutations can appear by chance or because of the $P$. aeruginosa adaptation for growing in MHB. Consequently, only those mutations present in the populations evolving under antibiotic selective pressure and not in the control populations evolving in absence of selection were taken into consideration. In other words, only those mutations that are enriched (and hence have been selected) under antibiotic selective pressure were taken into consideration. Indeed, all mutant alleles selected in the presence of antibiotics and not present in the populations grown without antibiotics present always a coverage $>50 \%$ and typically $>90 \%$ (Supplementary Table S1) in the whole population, indicating that they are under positive selection when $P$. aeruginosa grows in presence of antibiotics. Supplementary Table S1 shows the locations of all 35 confirmed genetic changes potentially associated with the development of resistance. A total of 31 single-nucleotide variants (SNVs) and 4 multi-nucleotide variants (MNVs; deletions and substitutions of various nucleotides and one transposition) were found, 31 located in genes and 4 in intergenic regions. The majority of the 


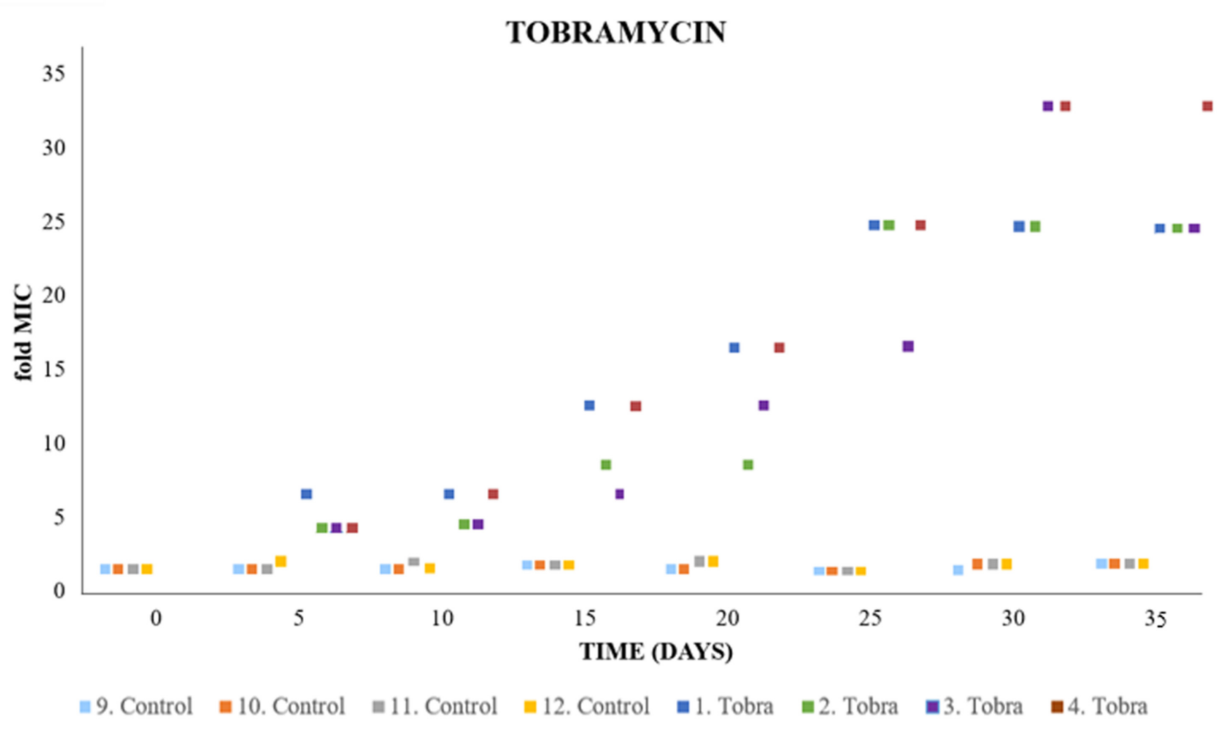

TIGECYCLINE

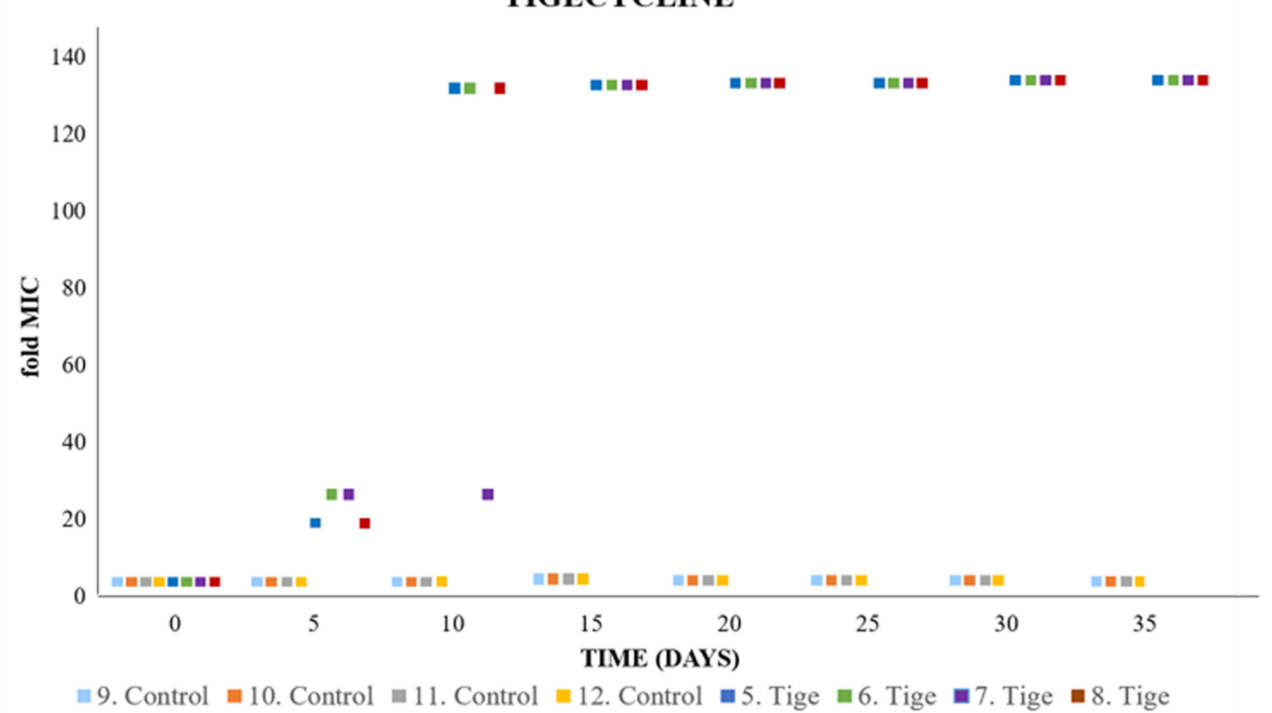

FIGURE 1 | Evolution of $P$. aeruginosa under antibiotic selective pressure. Graphs show the rise of the MICs over the evolution period, from the value corresponding to the wild-type strain (tobramycin [Tobra], $1 \mu \mathrm{g} / \mathrm{ml}$; tigecycline [Tige], $2 \mu \mathrm{g} / \mathrm{ml}$ ) to high levels of tobramycin/tigecycline resistance (doubling the antibiotic concentration every 5 days). The detection limit of the tigecycline $E$-test was $256 \mu \mathrm{g} / \mathrm{ml}$, limiting the assessment of resistance levels from day 15 to the end of the experiment. MIC values for each replicate are provided in Supplementary Table S3.

mutations located in genes resulted in amino acid alterations, frameshifts or stop codons. In addition, the coverage of the obtained reads was mapped to the $P$. aeruginosa genome to search for gene amplifications and deletions. One $377 \mathrm{bp}$ deletion was detected in the tigecycline-treated population 8 , comprising part of the gene coding for the transcriptional repressor of the multidrug efflux pump mexCD-oprJ, $n f x B$, plus a small region of the adjacent gene morA.

To further verify the presence in the evolved populations of the mutations identified by WGS (Supplementary Table S1), the regions containing these mutations were amplified and the amplicons Sanger-sequenced.

\section{Common Aspects of the Evolution of $P$. aeruginosa Toward Resistance to Tobramycin or Tigecycline}

The present results shed light on the development of resistance to ribosome-targeting antibiotics in $P$. aeruginosa. Although this opportunistic pathogen is already intrinsically resistant to 
TABLE 1 | MICs $(\mu \mathrm{g} / \mathrm{ml})$ of antibiotics of different structural families in the populations evolved at 32MIC tobramycin and tigecycline.

\begin{tabular}{|c|c|c|c|c|c|c|c|c|c|c|c|c|}
\hline Replicate & Tgc & Tet & Atm & Caz & Cip & Tob & $\mathbf{s}$ & Ak & C & $\mathbf{F}$ & Cs & PB \\
\hline PA14 & 1.5 & 16 & 1.5 & 1 & 0.094 & 1 & 16 & 2 & 24 & 24 & 1.5 & 0.75 \\
\hline \multicolumn{13}{|c|}{ Tobramycin 32MIC } \\
\hline 1 & 64 & 48 & 3 & 1.5 & 0.5 & 32 & 192 & $\geq 256$ & 24 & 1 & 6 & 2.5 \\
\hline 2 & 24 & 32 & 4 & 1.5 & 0.5 & 24 & 256 & 192 & 32 & 1 & 6 & 2.5 \\
\hline 3 & 12 & 24 & 4 & 1.5 & 0.19 & 8 & 64 & 128 & 24 & 1 & 4 & 3 \\
\hline 4 & 32 & 24 & 3 & 1.5 & 0.5 & 32 & 192 & $\geq 256$ & 24 & 1.5 & 5 & 2.5 \\
\hline \multicolumn{13}{|c|}{ Tigecycline 32MIC } \\
\hline 5 & $\geq 256$ & 192 & 8 & 4 & 0.75 & 4 & 96 & 32 & 128 & 2 & 2.5 & 1 \\
\hline 6 & $\geq 256$ & 192 & 8 & 3 & 0.5 & 1.5 & 64 & 16 & 64 & 1.5 & 2.5 & 1 \\
\hline 7 & $\geq 256$ & $\geq 256$ & 6 & 3 & 0.25 & 2 & 128 & 16 & 96 & 1 & 5 & 2 \\
\hline 8 & $\geq 256$ & 192 & 6 & 2 & 0.75 & 8 & 192 & 32 & 128 & 2 & 5 & 1.5 \\
\hline \multicolumn{13}{|c|}{ Controls } \\
\hline 9 & 2 & 12 & 2 & 1.5 & 0.094 & 1 & 12 & 4 & 24 & 32 & 1.5 & 0.75 \\
\hline 10 & 2 & 8 & 2 & 1 & 0.19 & 1 & 12 & 2 & 16 & 32 & 1.5 & 0.75 \\
\hline 11 & 1.5 & 12 & 2 & 1 & 0.094 & 1 & 12 & 3 & 32 & 32 & 1.5 & 1 \\
\hline 12 & 2 & 12 & 2 & 1.5 & 0.125 & 0.75 & 12 & 3 & 16 & 32 & 1.5 & 0.75 \\
\hline
\end{tabular}

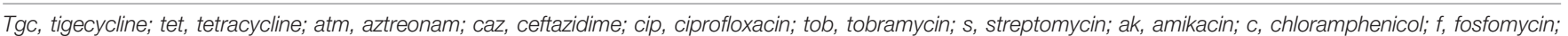
cs, colistin; pb, polymyxin B. All MICs were obtained by E-test, excepting for polymyxin B and colistin, which were analyzed via double dilution in a microtiter plate.

TABLE 2 | Previously described role on antibiotic resistance of genes presenting mutations in the evolved populations.

\begin{tabular}{|c|c|c|c|c|}
\hline Gene name & Tobramycin replicates & Tigecycline replicates & Previously described role in resistance & References \\
\hline pmrB & 4 & 2 & $\begin{array}{l}\text { Cationic peptides, quinolones, tigecycline, } \\
\text { tobramycin }\end{array}$ & $\begin{array}{l}\text { Muller et al., 2011; Moskowitz et al., 2012; } \\
\text { Lopez-Causape et al., 2018b }\end{array}$ \\
\hline fusA & 4 & - & Aminoglycosides & $\begin{array}{l}\text { Wang et al., 2015; Feng et al., 2016; Bolard } \\
\text { et al., 2018; Lopez-Causape et al., 2018b }\end{array}$ \\
\hline$p t s P$ & 2 & - & Tobramycin & Schurek et al., 2008 \\
\hline $\operatorname{mex} C D$ & - & 2; 1 mexC, 1 mexD & $\begin{array}{l}\text { Quinolones, tetracyclines, } \beta \text {-lactams, } \\
\text { chloramphenicol }\end{array}$ & Masuda et al., 2000b \\
\hline PA14_00180 & - & 2 & - & - \\
\hline rpsj & - & 2 & Tetracycline, tigecycline & Hu et al., 2005; Ahn et al., 2016 \\
\hline
\end{tabular}

tigecycline, highly resistant populations with MICs several times higher than that seen for the wild-type strain were selected for in the experimental evolution assays (Figure 1).

Overall, the results indicate that increased resistance to tigecycline and tobramycin comes about via distinguishable evolutionary trajectories but which show some similarities (Table 2). In particular, mutations in orf $N$ (selected in all eight replicates) and $p m r B$ (selected in 6 out of 8 replicates) were selected under pressure from either antibiotic (Figure 2). It should be noted that all populations evolved in the presence of antibiotic, and none of those evolved in unexposed control populations show mutants in orf $N$ during the first step in evolution (i.e., after the first change in antibiotic concentration). This gene codes for a putative glycosyl transferase needed for the glycosylation of type A flagellins (Schirm et al., 2004). Six of the studied mutants carried a single base pair deletion in a poly-G repeat in $\operatorname{orf} \mathrm{N}$, and two contained a single base pair insertion in the same region (Supplementary Table S1), all of them leading to a Val50fs mutation. Similar mutations have been found in $P$. aeruginosa when exposed to ciprofloxacin under experimental evolution conditions (Wong et al., 2012). The fact that these mutations are located in a poly-G repeat region supports that this gene might have a specific high mutation rate as the consequence of polymerase slippage (Moxon et al., 2006). We hypothesize that this might be the reason why orf $N$ mutants are detected in all replicates.

pmrAB is a two-component system involved in polymyxin resistance (Moskowitz et al., 2012). The system regulates the 


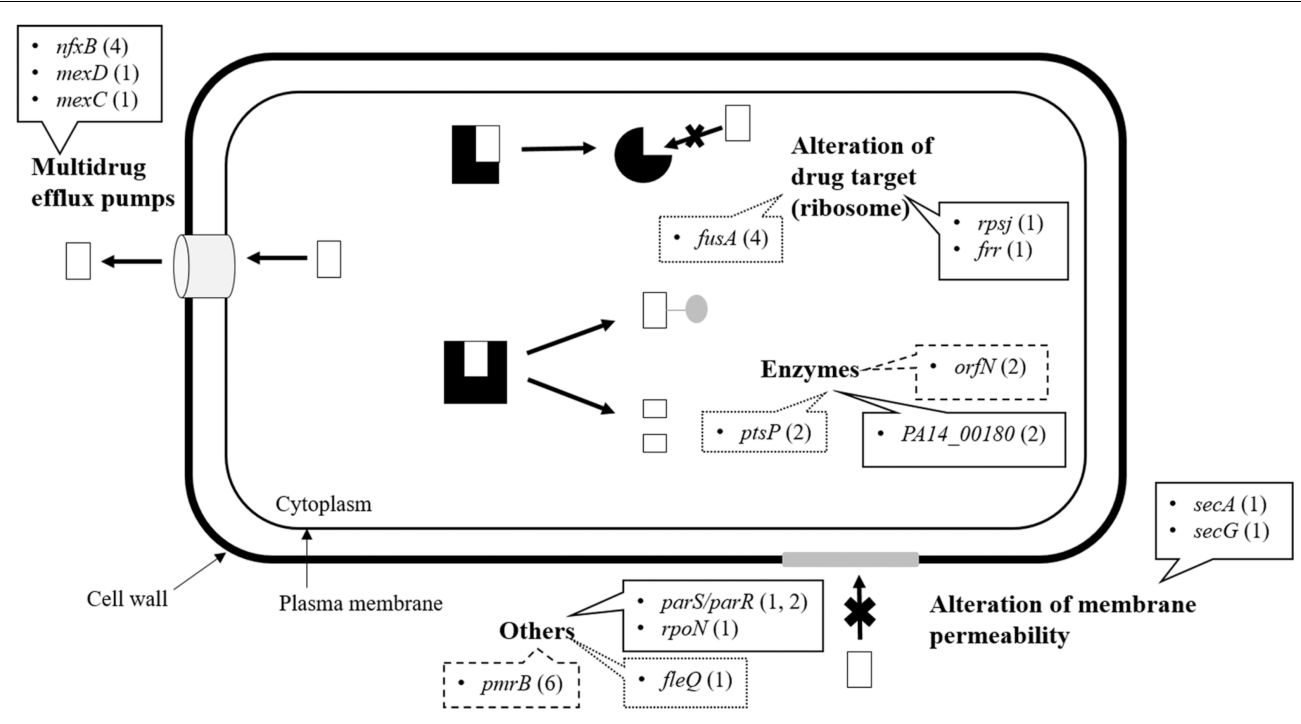

FIGURE 2 | Common and different genetic changes identified by whole-genome sequencing. Mutations detected by Illumina and verified by Sanger Sequencing in evolved $P$. aeruginosa PA14 at 32MIC tigecycline/tobramycin. Most mutations fell into the four major functional groups of resistance mechanism: multidrug efflux pumps, alteration of drug target, enzyme neutralization, and alteration of membrane permeability. Others that did not properly match any of these categories were entered the group "Others." The line type indicates the drug providing the selection pressure: continuous line, tigecycline; dots, tobramycin; dashes, both treatments. The figures after the genes refer to the number of different genetic changes found in each. These values also inform about the number of biological replicates in which a particular gene mutated, with the exceptions of rpsJ (only one type of mutation) and orfN (two different mutations that appeared in every evolved population).

expression of operons involved in the biosynthesis of lipid A with 4-aminoarabinose, which produces a more positively charged lipopolysaccharide, thus reducing the binding and the activity of cationic peptides (Gunn et al., 2000). Gain-of-function mutants with lipid A modifications can be selected for and are resistant to colistin (Fernández et al., 2010). Moreover, a recent evolution study of $P$. aeruginosa involving colistin gave rise to the selection of mutations in $p m r B$ (Jochumsen et al., 2016), and it has been proposed that mutations in such regulatory elements may potentiate the effect of other mutations (Lind et al., 2015). Indeed, $p m r B$ is involved in a wide range of antibiotic resistances, including those to quinolones, tigecycline, tobramycin and cationic peptides (Muller et al., 2011; Lopez-Causape et al., 2018 b). In agreement with these data, the populations selected in our evolution experiment present a reduced susceptibility to colistin and polymixin B (Table 1).

\section{Evolutionary Pathways Toward Tobramycin Resistance in $P$. aeruginosa}

Having shown the common features of the evolutionary trajectories toward resistance to both test antibiotics, the specific pathways toward resistance to each were sought. Mutations in fus $A$ and $p t s P$ were found to be involved in evolution toward tobramycin resistance (Schurek et al., 2008; Wang et al., 2015; Feng et al., 2016). Together with the above mentioned orf $N$ mutants, mutations in fusA, which codes for elongation factor $G$, may be envisaged as a first response to aminoglycosides (Wang et al., 2015; Feng et al., 2016) since they were observed in all evolved populations (Figure 2). Further, recent works have shown this type of mutations to be present in clinical isolates as well as in in vitro selected aminoglycoside resistant mutants (Bolard et al., 2018; Ibacache-Quiroga et al., 2018; LopezCausape et al., 2018b), reinforcing its role in the acquisition of aminoglycosides resistance. Although mexXY overexpression is considered a hallmark of aminoglycoside resistance development of CF chronic infections (Guenard et al., 2014), our data using PA14, as well as a recent experimental study using PAO1 (LopezCausape et al., 2018b), show that this does not necessarily always occurs, at least in vitro.

Consistent with a proposed role of pts $P$ in low-level tobramycin resistance (Schurek et al., 2008), the present results indicate that its mutation might also be important, because of its presence in 2 out of 4 tobramycin replicates. In fact, the MIC clearly increased when this mutation appeared alone (population 2, 8-24 $\mu \mathrm{g} / \mathrm{ml}$; Figure 3 and Supplementary Table S3). Whether or not this type of mutation is selected for in clinical settings deserves further investigation. Finally, FleQ is a major flagellar regulator that has been found responsive to c-di-GMP and plays a role in biofilm formation (Hickman and Harwood, 2008; Jimenez et al., 2012; Matsuyama et al., 2016), although it has not been previously described to be involved in antibiotic resistance in planktonic cells.

It has been previously shown that mutations in at least 135 genes can render low-level aminoglycoside resistance in $P$. aeruginosa (Schurek et al., 2008). Despite the large number of possible combinations that might be selected along evolution under antibiotic selective pressure, we found that all four evolved populations contained mutants in orf $N$, fus $A$ and $p m r B$ and two of these evolved populations also presented mutants in $p t s P$. Further, recent work performed independently in another laboratory has also shown that $f u s A$ and $p m r B$ mutants are 
A

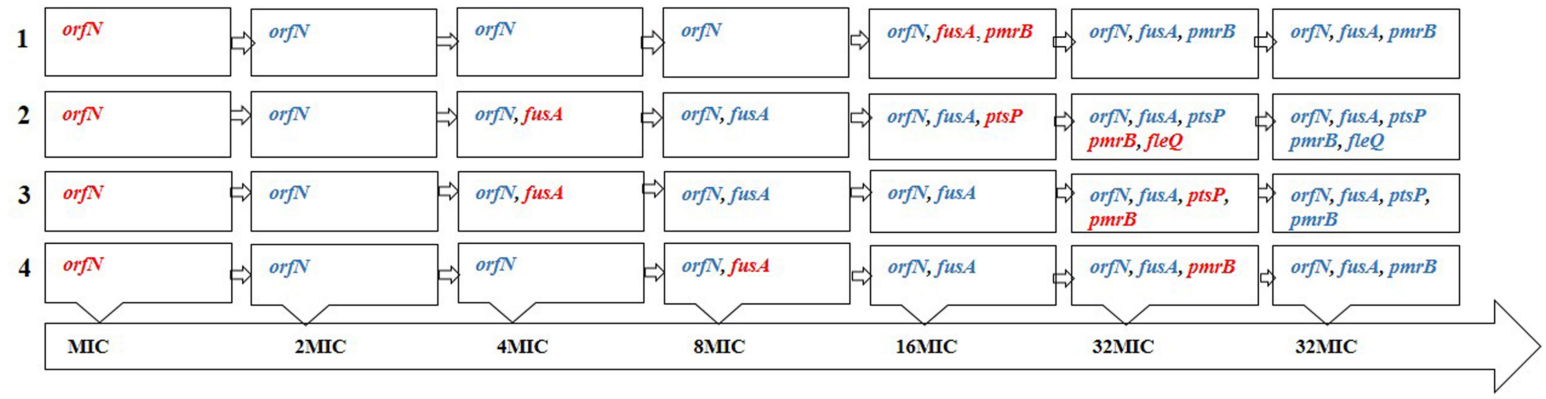

B

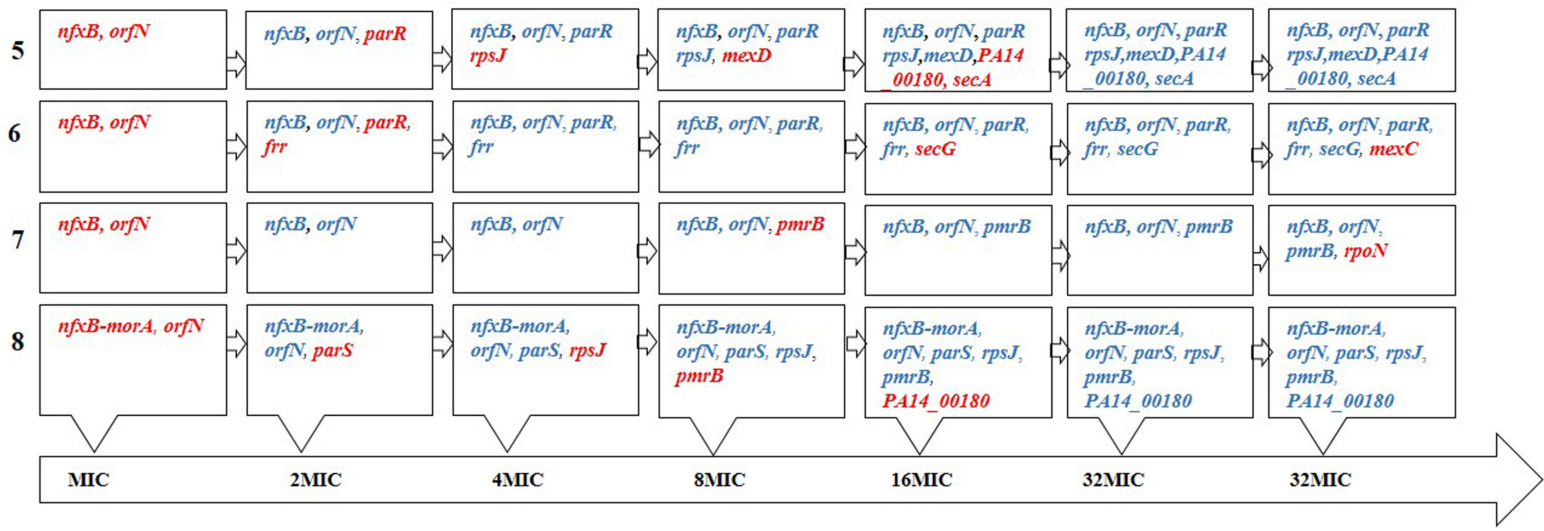

FIGURE 3 | Order of appearance of genetic changes. Order of appearance of (A) tobramycin and (B) tigecycline resistance mutations during the evolution process, as determined by PCR amplifications of known SNVs/MNVs in 32MIC populations. The names of the genes in red indicate that these mutations appeared in this step. Once a mutation appears it remains in the population until the end of the evolutionary period. We cannot discard that other mutations may have appeared and not fixed over the 35 days evolution period.

frequently selected in presence of aminoglycosides (LopezCausape et al., 2018b). Taking into consideration that the number of possible combinations of five mutations from the above mentioned 135 genes exceeds 3.4E8, our results indicate that $P$. aeruginosa mutation-driven resistance to tobramycin displays a certain degree of predictability.

\section{Evolutionary Pathways Toward Tigecycline Resistance in $P$. aeruginosa}

Pseudomonas aeruginosa PA14 evolving under selective pressure from tigecycline accumulated resistance mutations, indicating that, even in intrinsically resistant microorganisms, resistance may increase. Among the selected mutated genes, three rpsJ, parRS and $n f x B$ - have already been described involved in antibiotic resistance (a result that validates the present experimental strategy). The Val57 change in the $30 \mathrm{~S}$ ribosomal protein $\mathrm{S} 10$ (rpsJ), which was present in two out of four replicates, is commonly seen in tetracycline-resistant clinical isolates of pathogens such as Neisseria gonorrhoeae, (Hu et al., 2005) while in Klebsiella pneumoniae different mutations in this gene have been related to tigecycline resistance (Ahn et al., 2016). Further, the two-component parRS system has been extensively examined in association with resistance to various drugs. In our experimental evolution assay, three out of the four tigecycline evolved populations presented mutations in this two-component system (Figure 3), which may be indicative of its importance in resistance. ParRS may promote multidrug resistance via three mechanisms: by inducing the expression of efflux pumps (such as MexXY-OprM), by repressing the expression of porins, and via LPS alteration (Muller et al., 2011).

The transcriptional repressor of the multidrug efflux pump MexCD-OprJ (Purssell and Poole, 2013), one of the most clinically important of all efflux pumps in $P$. aeruginosa, is coded for by $n f x B$. Loss-of-function mutations in this gene result in the pump's overexpression. Different mutations in $n f x B$ were selected in all four replicates of the evolving populations, and most were fully inactivating mutations (a 377 bp deletion leading to a truncated protein; a transposition; and an $11 \mathrm{bp}$ deletion). Although it has been suggested that the main efflux pump contributing to tigecycline resistance in $P$. aeruginosa is MexXY-OprM (Masuda et al., 2000a; Dean et al., 2003), the first step along the path to resistance in the present work was seen to be the selection of mutants able to extrude tigecycline via MexCD-OprJ. To ascertain whether or not $n f x B$ selected mutations inactivate the repressor, hence allowing MexCD overexpression, one individual clone from each 5 days tigecycline evolved population was isolated in MHA. The presence of their corresponding $n f x B$ mutations and the absence of $\operatorname{orf} N$ modification were confirmed by Sanger-sequencing. Expression 


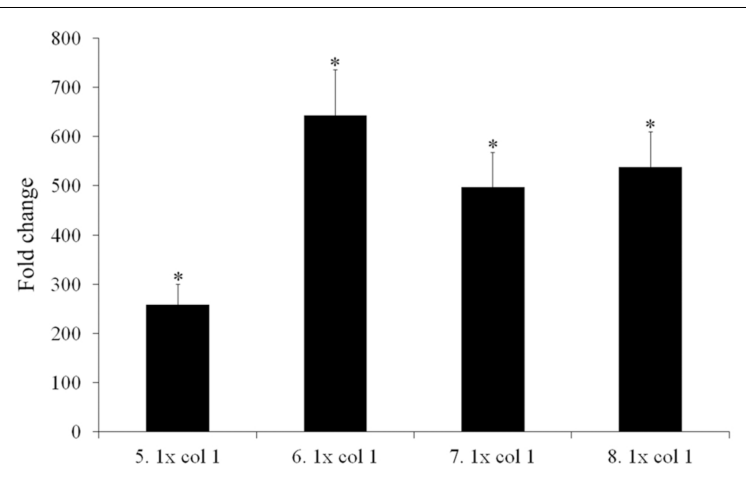

FIGURE 4 | mexC over-expression in tigecycline evolved individual clones. Four individual clones from the tigecycline evolved populations after 5 days of the experiment were isolated. The presence of the different $n f x B$ mutations, as well as the absence of orfN mutations, were addressed. Fold changes were estimated with respect to the value given by $P$. aeruginosa PA14 strain. Error bars indicate standard deviations of the results from three independent experiments. Statistically significant differences $(p<0.05)$ in the level of expression of each population with respect to the wild type strain was evaluated using the Student's $T$-test and are highlighted with asterisks. 5.1-8.1: clones isolated from the populations evolved under tigecycline selective pressure at day five of evolution.

of mexC was measured in comparison with the one of the wildtype strain. As shown in Figure 4, all clones carrying $n f x B$ mutations overexpressed mex $C$, confirming that these mutations inactivate, in different degree, the $\mathrm{NfxB}$ repressor. Two further mutations in each of the genes coding for the subunits of this efflux pump (mexC and mexD) were then selected for in populations 5 and 6 respectively. It may be that these mutations alter the specificity of MexCD-OprJ improving the capacity of the pump to extrude tigecycline. The MexD SNV (Phe608Cys) is located in one of the two large periplasmic loops known to be involved in substrate specificity (Elkins and Nikaido, 2002). In agreement with this, it has been described that mutations in AcrB, the Enterobacteriaceae ortholog of MexD, alter the pump's substrate profile (Blair et al., 2015). While a role for these mutations in reducing the susceptibility to tigecycline is a compelling hypothesis, it cannot be ruled out that mutations in mexD or mexC may compensate for any increased nonphysiological extrusion of some important cellular metabolite by those mutants that overexpress this pump.

The present results indicate that tigecycline resistance might also come about through changes in the Sec system ( SecA and $\operatorname{Sec} G$ ), likely via the modification of the permeability of the membrane and the impairment of tigecycline uptake. It has also been suggested that the Sec pathway could be involved in the translocation of components of multidrug efflux pumps across the bacterial membranes (Yoneyama et al., 2010; Akiba et al., 2013; Chaudhary et al., 2015), but it is unclear how changes in this translocation could increase resistance in the selected mutants. Tigecycline increased resistance may also come about through modifications of the $\sigma_{54}$ RpoN factor that has been found to modulate $P$. aeruginosa virulence (Kazmierczak et al., 2005), bacterial tolerance to carbapenems (Viducic et al., 2016), susceptibility to quinolones (Viducic et al., 2007) and survival in the presence of tobramycin (Viducic et al., 2017). Both the Sec pathway and RpoN have been proposed as excellent targets in the search of novel antibiotics (Jin et al., 2016; Lloyd et al., 2017). Our findings suggest that tigecyline might select mutants presenting cross-resistance to these potential inhibitors, still under development. Mutations in the gene encoding the ribosome recycling factor frr are likely related to modifications in the tigecycline target (ribosome). Indeed, this particular mutation is located in the start codon (Supplementary Table S1), so it may affect the level of $f r r$, impairing the steady state amount of active ribosomes. A similar situation might arise with PA14_00180, which codes for a putative rRNA small subunit methyltransferase, hence likely able to modify the ribosome.

All the changes seen during the evolution of the populations subjected to selective pressure from tigecycline indicate that evolutionary pathways toward tigecycline resistance present some common features. All four replicates present mutations in $\operatorname{orf} N$ and $n f x B$ and mutations in parRS were selected in three out of the four populations. Mutations at $\sec A G$, PA14_00180, rpsJ or mexCD were selected in half (two) of the populations. This indicates that $P$. aeruginosa can develop resistance to tigecycline by following a limited number of different evolutionary trajectories, which share some specific type of mutations, suggesting a certain degree of determinism.

In addition of conferring resistance to the selecting antibiotics, the respective mutants also showed resistance to antibiotics belonging to other structural categories and with different targets. This might be explained, at least in part, via the important role that efflux pumps seem to play in the development of resistance. Indeed, all mutants selected in the presence of tigecycline showed mutations in $n f x B$, which would lead to the overexpression of MexCD-OprJ. In turn this might reduce susceptibility to quinolones, tetracyclines, beta-lactams and chloramphenicol (De Kievit et al., 2001) - phenotypes in agreement with the present data (Table 1). Moreover, all four population replicates analyzed showed reduced susceptibility to aminoglycosides. This phenotype may be explained in three populations as a result of the mutations selected for in the ParRS system, which is involved in modification of the lipopolysaccharide and in the regulation of the expression of MexXY-OprM, an efflux pump that contributes to aminoglycoside resistance in $P$. aeruginosa (Masuda et al., 2000a), and which is described as being regulated by the two-component system ParRS (Muller et al., 2011).

\section{CONCLUSION}

Experimental evolution approaches may allow determining basic aspects of evolution, among which knowing to what extent evolution (in our case of asexual organisms) can be predictable and hence deterministic or is basically stochastic (non-predictable) (Lassig et al., 2017), is notably interesting. This is particularly relevant in the case of antibiotic resistance, a field in which the implementation of novel therapeutic approaches is based in the assumption that evolution of antibiotic resistance can be largely predictable (Martinez et al., 2007), a feature that goes against most common views on evolution. 
The present work provides information on the evolutionary trajectories leading to resistance to antibiotics belonging to different structural families but targeting the ribosome in $P$. aeruginosa. The results suggest that, although mutations in several genes may contribute to the development of antibiotic resistance (Fajardo et al., 2008; Tamae et al., 2008; AlvarezOrtega et al., 2011; Martinez, 2012; Vestergaard et al., 2016; Lopez-Causape et al., 2018a), which may imply a large degree of stochasticity in the evolutionary trajectories, mutation-driven evolution toward resistance is partially deterministic, at least when bacteria grow in the same conditions. This opens up the possibility of predicting the appearance of antibiotic resistance (Martinez et al., 2007).

The selection of specific mutations depends primarily on the fitness of each mutant before/after selection and the rate of mutation supply for each of the mutations (Huseby et al., 2017; Hughes and Andersson, 2017). Recent work has shown that a high generalized mutation supply does not alter the types of mutations selected upon experimental evolution (IbacacheQuiroga et al., 2018), in which case fitness costs, together with population bottlenecks (Vogwill et al., 2016), will be the main constrains for selecting some mutants over other mutations able of providing the same resistance phenotype. However, the situation might be different in the case of gene-specific high mutation rates, a situation that might have happened in the case of $\operatorname{orf} \mathrm{N}$. To note here that all mutations in $\operatorname{orf} \mathrm{N}$ have been selected in a poly-G region, present in the P. aeruginosa PA14 strain, a situation that might increase the mutation rate as the consequence of polymerase slippage (Moxon et al., 2006). We hypothesize that this gene-specific high mutational supply might be in the basis of the presence of orf $N$ mutants in all replicates. However, OrfN sequence is highly polymorphic in $P$. aeruginosa (Arora et al., 2004) becoming doubtful if these results can be extrapolated to strains with different $\operatorname{orf} \mathrm{N}$ alleles.

The present results clearly show that challenging $P$. aeruginosa with tigecycline has clinically relevant consequences, despite this bacterial pathogen is considered to be intrinsically resistant to this antibiotic. Tigecycline is used for empiric treatment of Gram-negative infections, and in this type of patients, the main agent causing superinfection is P. aeruginosa (GarciaCabrera et al., 2010; Ulu-Kilic et al., 2015; Katsiari et al., 2016), which is most likely under tigecycline selection during treatment. Our results indicate that tigecycline selects mutants presenting reduced susceptibility to antibiotics of clinical value as aztreonam, ceftazidime, ciprofloxacin or aminoglycosides. Notably, these mutants also display an increased susceptibility to fosfomycin. Our results suggest that fosfomycin might be an antibiotic of choice for treating superinfections by $P$. aeruginosa in tigecycline treated patients. A recent work has shown that

\section{REFERENCES}

Ahn, C., Yoon, S. S., Yong, T. S., Jeong, S. H., and Lee, K. (2016). The resistance mechanism and clonal distribution of tigecycline-nonsusceptible Klebsiella pneumoniae isolates in Korea. Yonsei Med. J. 57, 641-646. doi: 10.3349/ymj. 2016.57.3.641 most antibiotic resistance mutations display strain-independent phenotypes (Knopp and Andersson, 2018). Nevertheless, it is also true that the current work has been performed with a single, model strain and, because of the diversity of $P$. aeruginosa, it would be important to examine additional strains to establish whether this phenotype depends on the genomic context or can be extrapolated to other $P$. aeruginosa clinical isolates.

Antibiotic resistance can be acquired by the acquisition of antibiotic resistance genes, which usually confer resistance to members of the same family of antibiotics. However, particularly relevant in the case of chronic infections is the selection of antibiotic resistant mutants. Our results indicate that antibiotic resistance mutations frequently have a pleiotropic effect, altering susceptibility to other drugs. In agreement with this is the fact that some of the mutations selected for by exposure to tobramycin or tigecycline have previously been described to confer resistance to other antibiotics belonging to different structural families.

\section{AUTHOR CONTRIBUTIONS}

FS-G and SH-A performed the experiments. JM and SH-A designed the work. All the authors participated in the interpretation of the results and in writing the article.

\section{FUNDING}

Work in our laboratory is supported by grants from the Instituto de Salud Carlos III (Spanish Network for Research on Infectious Diseases [RD16/0016/0011]), from the Spanish Ministry of Economy and Competitiveness (BIO2017-83128-R and BIO2014-54507-R) CNB, and from the Autonomous Community of Madrid (B2017/BMD-3691). FS-G is the recipient of an FPU fellowship from the Spanish Ministry of Education. The funders had no role in study design, data collection and interpretation, or the decision to submit the work for publication.

\section{ACKNOWLEDGMENTS}

We thank Adrian Burton (www.physicalevidence.es) for editing the manuscript.

\section{SUPPLEMENTARY MATERIAL}

The Supplementary Material for this article can be found online at: https://www.frontiersin.org/articles/10.3389/fgene. 2018.00451/full\#supplementary-material 
aeruginosa to beta-lactam antibiotics. Antimicrob. Agents Chemother. 54, 41594167. doi: 10.1128/AAC.00257-10

Alvarez-Ortega, C., Wiegand, I., Olivares, J., Hancock, R. E., and Martinez, J. L. (2011). The intrinsic resistome of Pseudomonas aeruginosa to beta-lactams. Virulence 2, 144-146.

Appelbaum, P. C. (2012). 2012 and beyond: potential for the start of a second pre-antibiotic era? J. Antimicrob. Chemother. 67, 2062-2068. doi: 10.1093/jac/ dks213dks213

Arora, S. K., Wolfgang, M. C., Lory, S., and Ramphal, R. (2004). Sequence polymorphism in the glycosylation island and flagellins of Pseudomonas aeruginosa. J. Bacteriol. 186, 2115-2122.

Blair, J. M., Bavro, V. N., Ricci, V., Modi, N., Cacciotto, P., Kleinekathfer, U., et al. (2015). AcrB drug-binding pocket substitution confers clinically relevant resistance and altered substrate specificity. Proc. Natl. Acad. Sci. U.S.A. 112, 3511-3516. doi: 10.1073/pnas.1419939112

Blake, K. L., and O’Neill, A. J. (2013). Transposon library screening for identification of genetic loci participating in intrinsic susceptibility and acquired resistance to antistaphylococcal agents. J. Antimicrob. Chemother. 68, 12-16. doi: 10.1093/jac/dks373

Bolard, A., Plesiat, P., and Jeannot, K. (2018). Mutations in gene fusA1 as a novel mechanism of aminoglycoside resistance in clinical strains of Pseudomonas aeruginosa. Antimicrob. Agents Chemother. 62:e1835-17. doi: 10.1128/aac. 01835- 17

Brandis, G., Pietsch, F., Alemayehu, R., and Hughes, D. (2015). Comprehensive phenotypic characterization of rifampicin resistance mutations in Salmonella provides insight into the evolution of resistance in Mycobacterium tuberculosis. J. Antimicrob. Chemother. 70, 680-685. doi: 10.1093/jac/dku434

Breidenstein, E. B., Khaira, B. K., Wiegand, I., Overhage, J., and Hancock, R. E. (2008). Complex ciprofloxacin resistome revealed by screening a Pseudomonas aeruginosa mutant library for altered susceptibility. Antimicrob. Agents Chemother. 52, 4486-4491. doi: 10.1128/AAC.00222-08

Bryson, V., and Szybalski, W. (1952). Microbial selection. Science 116, 45-51. doi: $10.1126 /$ science.116.3003.45

Cabot, G., Zamorano, L., Moya, B., Juan, C., Navas, A., Blazquez, J., et al. (2016). Evolution of Pseudomonas aeruginosa antimicrobial resistance and fitness under low and high mutation rates. Antimicrob. Agents Chemother. 60, 1767-1778. doi: 10.1128/AAC.02676-15

Chaudhary, A. S., Chen, W., Jin, J., Tai, P. C., and Wang, B. (2015). SecA: a potential antimicrobial target. Future Med. Chem. 7, 989-1007. doi: 10.4155/fmc. 15.42

Cheer, S. M., Waugh, J., and Noble, S. (2003). Inhaled tobramycin (TOBI): a review of its use in the management of Pseudomonas aeruginosa infections in patients with cystic fibrosis. Drugs 63, 2501-2520.

De Kievit, T. R., Parkins, M. D., Gillis, R. J., Srikumar, R., Ceri, H., Poole, K., et al. (2001). Multidrug efflux pumps: expression patterns and contribution to antibiotic resistance in Pseudomonas aeruginosa biofilms. Antimicrob. Agents Chemother. 45, 1761-1770.

Dean, C. R., Visalli, M. A., Projan, S. J., Sum, P. E., and Bradford, P. A. (2003). Efflux-mediated resistance to tigecycline (GAR-936) in Pseudomonas aeruginosa PAO1. Antimicrob. Agents Chemother. 47, 972-978.

Elkins, C. A., and Nikaido, H. (2002). Substrate specificity of the RND-type multidrug efflux pumps AcrB and AcrD of Escherichia coli is determined predominantly by two large periplasmic loops. J. Bacteriol. 184, 6490-6498.

Fajardo, A., Martinez-Martin, N., Mercadillo, M., Galan, J. C., Ghysels, B., Matthijs, S., et al. (2008). The neglected intrinsic resistome of bacterial pathogens. PLoS One 3:e1619. doi: 10.1371/journal.pone.0001619

Feng, Y., Jonker, M. J., Moustakas, I., Brul, S., and Ter Kuile, B. H. (2016). Dynamics of mutations during development of resistance by Pseudomonas aeruginosa against five antibiotics. Antimicrob. Agents Chemother. 60, 4229-4236. doi: 10.1128/AAC.00434-16

Fernandez, L., Alvarez-Ortega, C., Wiegand, I., Olivares, J., Kocincova, D., Lam, J. S., et al. (2013). Characterization of the polymyxin B resistome of Pseudomonas aeruginosa. Antimicrob. Agents Chemother. 57, 110-119. doi: 10. 1128/AAC.01583-12

Fernández, L., Gooderham, W. J., Bains, M., McPhee, J. B., Wiegand, I., and Hancock, R. E. (2010). Adaptive resistance to the "last hope" antibiotics polymyxin B and colistin in Pseudomonas aeruginosa is mediated by the novel two-component regulatory system ParR-ParS. Antimicrob. Agents Chemother. 54, 3372-3382. doi: 10.1128/AAC.00242-10
Garcia-Cabrera, E., Jimenez-Mejias, M. E., Gil Navarro, M. V., Gomez-Gomez, M. J., Ortiz-Leyba, C., Cordero, E., et al. (2010). Superinfection during treatment of nosocomial infections with tigecycline. Eur. J. Clin. Microbiol. Infect. Dis. 29, 867-871. doi: 10.1007/s10096-010-0942-y

Guenard, S., Muller, C., Monlezun, L., Benas, P., Broutin, I., Jeannot, K., et al. (2014). Multiple mutations lead to MexXY-OprM-dependent aminoglycoside resistance in clinical strains of Pseudomonas aeruginosa. Antimicrob. Agents Chemother. 58, 221-228. doi: 10.1128/aac.01252-13

Gunn, J. S., Ryan, S. S., Van Velkinburgh, J. C., Ernst, R. K., and Miller, S. I. (2000). Genetic and functional analysis of a PmrA-PmrB-regulated locus necessary for lipopolysaccharide modification, antimicrobial peptide resistance, and oral virulence of Salmonella enterica serovar typhimurium. Infect. Immun. 68, 6139-6146.

Hernando-Amado, S., Sanz-Garcia, F., Blanco, P., and Martinez, J. L. (2017). Fitness costs associated with the acquisition of antibiotic resistance. Essays Biochem. 61, 37-48. doi: 10.1042/ebc20160057

Hickman, J. W., and Harwood, C. S. (2008). Identification of FleQ from Pseudomonas aeruginosa as a c-di-GMP-responsive transcription factor. Mol. Microbiol. 69, 376-389. doi: 10.1111/j.1365-2958.2008.06281.x

Hu, M., Nandi, S., Davies, C., and Nicholas, R. A. (2005). High-level chromosomally mediated tetracycline resistance in Neisseria gonorrhoeae results from a point mutation in the rpsJ gene encoding ribosomal protein S10 in combination with the mtrR and penB resistance determinants. Antimicrob. Agents Chemother. 49, 4327-4334. doi: 10.1128/AAC.49.10.4327-4334. 2005

Hughes, D., and Andersson, D. I. (2017). Evolutionary trajectories to antibiotic resistance. Annu. Rev. Microbiol. 71, 579-596. doi: 10.1146/annurev-micro090816-093813

Huseby, D. L., Pietsch, F., Brandis, G., Garoff, L., Tegehall, A., and Hughes, D. (2017). Mutation supply and relative fitness shape the genotypes of ciprofloxacin-resistant Escherichia coli. Mol. Biol. Evol. 34, 1029-1039. doi: $10.1093 / \mathrm{molbev} / \mathrm{msx} 052$

Ibacache-Quiroga, C., Oliveros, J. C., Couce, A., and Blázquez, J. (2018). Parallel evolution of high-level aminoglycoside resistance in Escherichia coli under low and high mutation supply rates. Front. Microbiol. 9:427. doi: 10.3389/fmicb. 2018.00427

Jimenez, P. N., Koch, G., Thompson, J. A., Xavier, K. B., Cool, R. H., and Quax, W. J. (2012). The multiple signaling systems regulating virulence in Pseudomonas aeruginosa. Microbiol. Mol. Biol. Rev. 76, 46-65. doi: 10.1128/MMBR.05007-11

Jin, J., Hsieh, Y. H., Cui, J., Damera, K., Dai, C., Chaudhary, A. S., et al. (2016). Using chemical probes to assess the feasibility of targeting seca for developing antimicrobial agents against gram-negative bacteria. ChemMedChem 11, 2511-2521. doi: $10.1002 / \mathrm{cmdc} 201600421$

Jochumsen, N., Marvig, R. L., Damkiaer, S., Jensen, R. L., Paulander, W., Molin, S., et al. (2016). The evolution of antimicrobial peptide resistance in Pseudomonas aeruginosa is shaped by strong epistatic interactions. Nat. Commun. 7:13002. doi: $10.1038 /$ ncomms 13002

Katsiari, M., Ntorlis, K., Nteves, I., Roussou, Z., Platsouka, E. D., and Maguina, A. (2016). Characteristics of superinfections during treatment with tigecycline. J. Chemother. 28, 110-115. doi: 10.1080/1120009x.2015.1118184

Kazmierczak, M. J., Wiedmann, M., and Boor, K. J. (2005). Alternative sigma factors and their roles in bacterial virulence. Microbiol. Mol. Biol. Rev. 69, 527-543. doi: 10.1128/MMBR.69.4.527-543.2005

Knopp, M., and Andersson, D. I. (2018). Predictable phenotypes of antibiotic resistance mutations. mBio 9:e00770-18. doi: 10.1128/mBio.00770- 18

Kotra, L. P., Haddad, J., and Mobashery, S. (2000). Aminoglycosides: perspectives on mechanisms of action and resistance and strategies to counter resistance. Antimicrob. Agents Chemother. 44, 3249-3256.

Lassig, M., Mustonen, V., and Walczak, A. M. (2017). Predicting evolution. Nat. Ecol. Evol. 1:77. doi: 10.1038/s41559-017-0077

Levin, B. R., and Rozen, D. E. (2006). Non-inherited antibiotic resistance. Nat. Rev. Microbiol. 4, 556-562.

Lind, P. A., Farr, A. D., and Rainey, P. B. (2015). Experimental evolution reveals hidden diversity in evolutionary pathways. eLife 4:e07074. doi: 10.7554/eLife. 07074

Livak, K. J., and Schmittgen, T. D. (2001). Analysis of relative gene expression data using real-time quantitative PCR and the 2(-Delta Delta C(T)) Method. Methods 25, 402-408. 
Lloyd, M. G., Lundgren, B. R., Hall, C. W., Gagnon, L. B., Mah, T. F., Moffat, J. F., et al. (2017). Targeting the alternative sigma factor RpoN to combat virulence in Pseudomonas aeruginosa. Sci. Rep. 7:12615. doi: 10.1038/s41598-017-12667-y

Lopez-Causape, C., Cabot, G., Del Barrio-Tofino, E., and Oliver, A. (2018a). The versatile mutational resistome of Pseudomonas aeruginosa. Front. Microbiol. 9:685. doi: 10.3389/fmicb.2018.00685

Lopez-Causape, C., Rubio, R., Cabot, G., and Oliver, A. (2018b). Evolution of the Pseudomonas aeruginosa aminoglycoside mutational resistome in vitro and in the cystic fibrosis setting. Antimicrob. Agents Chemother. 62:e02583-17. doi: 10.1128/aac.02583-17

Martinez, J. L. (2012). The antibiotic resistome: challenge and opportunity for therapeutic intervention. Future Med. Chem. 4, 347-359. doi: 10.4155/fmc.12.2

Martinez, J. L., and Baquero, F. (2000). Mutation frequencies and antibiotic resistance. Antimicrob. Agents Chemother. 44, 1771-1777.

Martinez, J. L., Baquero, F., and Andersson, D. I. (2007). Predicting antibiotic resistance. Nat. Rev. Microbiol. 5, 958-965.

Martinez, J. L., Baquero, F., and Andersson, D. I. (2011). Beyond serial passages: new methods for predicting the emergence of resistance to novel antibiotics. Curr. Opin. Pharmacol. 11, 439-445. doi: 10.1016/j.coph.2011.07.005

Martinez, J. L., Coque, T. M., and Baquero, F. (2015). What is a resistance gene? Ranking risk in resistomes. Nat. Rev. Microbiol. 13, 116-123. doi: 10.1038/ nrmicro3399

Martinez, J. L., Fajardo, A., Garmendia, L., Hernandez, A., Linares, J. F., MartinezSolano, L., et al. (2009). A global view of antibiotic resistance. FEMS Microbiol. Rev. 33, 44-65. doi: 10.1111/j.1574-6976.2008.00142.x

Martinez, J. L., and Rojo, F. (2011). Metabolic regulation of antibiotic resistance. FEMS Microbiol. Rev. 35, 768-789. doi: 10.1111/j.1574-6976.2011.00282.x

Martinez-Solano, L., Macia, M. D., Fajardo, A., Oliver, A., and Martinez, J. L. (2008). Chronic Pseudomonas aeruginosa infection in chronic obstructive pulmonary disease. Clin. Infect. Dis. 47, 1526-1533. doi: 10.1086/593186

Masuda, N., Sakagawa, E., Ohya, S., Gotoh, N., Tsujimoto, H., and Nishino, T. (2000a). Contribution of the MexX-MexY-oprM efflux system to intrinsic resistance in Pseudomonas aeruginosa. Antimicrob. Agents Chemother. 44, 2242-2246.

Masuda, N., Sakagawa, E., Ohya, S., Gotoh, N., Tsujimoto, H., and Nishino, T. (2000b). Substrate specificities of MexAB-OprM, MexCD-OprJ, and MexXYoprM efflux pumps in Pseudomonas aeruginosa. Antimicrob. Agents Chemother. 44, 3322-3327.

Matsuyama, B. Y., Krasteva, P. V., Baraquet, C., Harwood, C. S., Sondermann, H., and Navarro, M. V. (2016). Mechanistic insights into c-di-GMP-dependent control of the biofilm regulator FleQ from Pseudomonas aeruginosa. Proc. Natl. Acad. Sci. U.S.A. 113, E209-E218. doi: 10.1073/pnas.1523148113

Meftahi, N., Namouchi, A., Mhenni, B., Brandis, G., Hughes, D., and Mardassi, H. (2016). Evidence for the critical role of a secondary site rpoB mutation in the compensatory evolution and successful transmission of an MDR tuberculosis outbreak strain. J. Antimicrob. Chemother. 71, 324-332. doi: 10.1093/jac/ dkv345

Moskowitz, S. M., Brannon, M. K., Dasgupta, N., Pier, M., Sgambati, N., Miller, A. K., et al. (2012). PmrB mutations promote polymyxin resistance of Pseudomonas aeruginosa isolated from colistin-treated cystic fibrosis patients. Antimicrob. Agents Chemother. 56, 1019-1030. doi: 10.1128/AAC.05 $829-11$

Moxon, R., Bayliss, C., and Hood, D. (2006). Bacterial contingency loci: the role of simple sequence DNA repeats in bacterial adaptation. Annu. Rev. Genet. 40, 307-333. doi: 10.1146/annurev.genet.40.110405.090442

Mulet, X., Maciá, M. D., Mena, A., Juan, C., Pérez, J. L., and Oliver, A. (2009). Azithromycin in Pseudomonas aeruginosa biofilms: bactericidal activity and selection of nfxB mutants. Antimicrob. Agents Chemother. 53, 1552-1560. doi: 10.1128/aac.01264-08

Muller, C., Plesiat, P., and Jeannot, K. (2011). A two-component regulatory system interconnects resistance to polymyxins, aminoglycosides, fluoroquinolones, and beta-lactams in Pseudomonas aeruginosa. Antimicrob. Agents Chemother. 55, 1211-1221. doi: 10.1128/aac.01252-10

Palmer, G. C., and Whiteley, M. (2015). Metabolism and pathogenicity of Pseudomonas aeruginosa infections in the lungs of individuals with cystic fibrosis. Microbiol. Spectr. 3. doi: 10.1128/microbiolspec.MBP-0003-2014

Pankey, G. A. (2005). Tigecycline. J. Antimicrob. Chemother. 56, 470-480. doi: $10.1093 /$ jac/dki248
Pietsch, F., Bergman, J. M., Brandis, G., Marcusson, L. L., Zorzet, A., Huseby, D. L., et al. (2017). Ciprofloxacin selects for RNA polymerase mutations with pleiotropic antibiotic resistance effects. J. Antimicrob. Chemother. 72, 75-84. doi: $10.1093 / \mathrm{jac} / \mathrm{dkw} 364$

Purssell, A., and Poole, K. (2013). Functional characterization of the NfxB repressor of the mexCD-oprJ multidrug efflux operon of Pseudomonas aeruginosa. Microbiology 159(Pt 10), 2058-2073. doi: 10.1099/mic.0.069286-0

Roca, I., Akova, M., Baquero, F., Carlet, J., Cavaleri, M., Coenen, S., et al. (2015). The global threat of antimicrobial resistance: science for intervention. New Microbes New Infect. 6, 22-29. doi: 10.1016/j.nmni.2015.02.007

Schirm, M., Arora, S. K., Verma, A., Vinogradov, E., Thibault, P., Ramphal, R., et al. (2004). Structural and genetic characterization of glycosylation of type a flagellin in Pseudomonas aeruginosa. J. Bacteriol. 186, 2523-2531.

Schurek, K. N., Marr, A. K., Taylor, P. K., Wiegand, I., Semenec, L., Khaira, B. K., et al. (2008). Novel genetic determinants of low-level aminoglycoside resistance in Pseudomonas aeruginosa. Antimicrob. Agents Chemother. 52, 4213-4219. doi: 10.1128/AAC.00507-08

Scortti, M., Lacharme-Lora, L., Wagner, M., Chico-Calero, I., Losito, P., and Vázquez-Boland, J. A. (2006). Coexpression of virulence and fosfomycin susceptibility in Listeria: molecular basis of an antimicrobial in vitro-in vivo paradox. Nat. Med. 12, 515-517.

Shcherbakov, D., Akbergenov, R., Matt, T., Sander, P., Andersson, D. I., and Böttger, E. C. (2010). Directed mutagenesis of Mycobacterium smegmatis $16 \mathrm{~S}$ rRNA to reconstruct the in-vivo evolution of aminoglycoside resistance in Mycobacterium tuberculosis. Mol. Microbiol. 77, 830-840. doi: 10.1111/j.13652958.2010.07218.x

Silby, M. W., Winstanley, C., Godfrey, S. A., Levy, S. B., and Jackson, R. W. (2011). Pseudomonas genomes: diverse and adaptable. FEMS Microbiol. Rev. 35, 652-680. doi: 10.1111/j.1574-6976.2011.00269.x

Tamae, C., Liu, A., Kim, K., Sitz, D., Hong, J., Becket, E., et al. (2008). Determination of antibiotic hypersensitivity among 4,000 single-geneknockout mutants of Escherichia coli. J. Bacteriol. 190, 5981-5988. doi: 10.1128/ JB.01982-07

Toprak, E., Veres, A., Michel, J. B., Chait, R., Hartl, D. L., and Kishony, R. (2011). Evolutionary paths to antibiotic resistance under dynamically sustained drug selection. Nat. Genet. 44, 101-105. doi: 10.1038/ng.1034

Tummler, B., Wiehlmann, L., Klockgether, J., and Cramer, N. (2014). Advances in understanding Pseudomonas. F1000Prime Rep. 6:9. doi: 10.12703/P6-9

Turrientes, M. C., Baquero, F., Levin, B. R., Martinez, J. L., Ripoll, A., GonzalezAlba, J. M., et al. (2013). Normal mutation rate variants arise in a Mutator (Mut S) Escherichia coli population. PLoS One 8:e72963. doi: 10.1371/journal.pone. 0072963

Ulu-Kilic, A., Alp, E., Altun, D., Cevahir, F., Kalın, G., and Demiraslan, H. (2015). Increasing frequency of Pseudomonas aeruginosa infections during tigecycline use. J. Infect. Dev. Ctries. 9, 309-312. doi: 10.3855/jidc.4700

Vestergaard, M., Leng, B., Haaber, J., Bojer, M. S., Vegge, C. S., and Ingmer, H. (2016). Genome-wide identification of antimicrobial intrinsic resistance determinants in Staphylococcus aureus. Front. Microbiol. 7:2018. doi: 10.3389/ fmicb.2016.02018

Viducic, D., Murakami, K., Amoh, T., Ono, T., and Miyake, Y. (2016). RpoN modulates carbapenem tolerance in Pseudomonas aeruginosa through Pseudomonas quinolone signal and PqsE. Antimicrob. Agents Chemother. 60, 5752-5764. doi: 10.1128/aac.00260-16

Viducic, D., Murakami, K., Amoh, T., Ono, T., and Miyake, Y. (2017). RpoN promotes Pseudomonas aeruginosa survival in the presence of tobramycin. Front. Microbiol. 8:839. doi: 10.3389/fmicb.2017.00839

Viducic, D., Ono, T., Murakami, K., Katakami, M., Susilowati, H., and Miyake, Y. (2007). rpoN gene of Pseudomonas aeruginosa alters its susceptibility to quinolones and carbapenems. Antimicrob. Agents Chemother. 51, 1455-1462. doi: 10.1128/AAC.00348-06

Vogne, C., Aires, J. R., Bailly, C., Hocquet, D., and Plesiat, P. (2004). Role of the multidrug efflux system MexXY in the emergence of moderate resistance to aminoglycosides among Pseudomonas aeruginosa isolates from patients with cystic fibrosis. Antimicrob. Agents Chemother. 48, 1676-1680.

Vogwill, T., Phillips, R. L., Gifford, D. R., and MacLean, R. C. (2016). Divergent evolution peaks under intermediate population bottlenecks during bacterial experimental evolution. Proc. Biol. Sci. 283:20160749. doi: 10.1098/rspb.2016. 0749 
Wang, D., Dorosky, R. J., Han, C. S., Lo, C. C., Dichosa, A. E., Chain, P. S., et al. (2015). Adaptation genomics of a small-colony variant in a Pseudomonas chlororaphis 30-84 biofilm. Appl. Environ. Microbiol. 81, 890-899. doi: 10.1128/ AEM.02617-14

Westbrock-Wadman, S., Sherman, D. R., Hickey, M. J., Coulter, S. N., Zhu, Y. Q., Warrener, P., et al. (1999). Characterization of a Pseudomonas aeruginosa efflux pump contributing to aminoglycoside impermeability. Antimicrob. Agents Chemother. 43, 2975-2983.

Wong, A., Rodrigue, N., and Kassen, R. (2012). Genomics of adaptation during experimental evolution of the opportunistic pathogen Pseudomonas aeruginosa. PLoS Genet. 8:e1002928. doi: 10.1371/journal.pgen.1002928

Yoneyama, H., Akiba, K., Hori, H., Ando, T., and Nakae, T. (2010). Tat pathwaymediated translocation of the sec pathway substrate protein MexA, an inner membrane component of the MexAB-OprM xenobiotic extrusion pump in Pseudomonas aeruginosa. Antimicrob. Agents Chemother. 54, 1492-1497. doi: 10.1128/aac.01495-09

Conflict of Interest Statement: The authors declare that the research was conducted in the absence of any commercial or financial relationships that could be construed as a potential conflict of interest.

Copyright (C) 2018 Sanz-García, Hernando-Amado and Martínez. This is an openaccess article distributed under the terms of the Creative Commons Attribution License (CC BY). The use, distribution or reproduction in other forums is permitted, provided the original author(s) and the copyright owner(s) are credited and that the original publication in this journal is cited, in accordance with accepted academic practice. No use, distribution or reproduction is permitted which does not comply with these terms. 